\title{
Optimal Surveillance of Intraductal Papillary Mucinous Neoplasms of the Pancreas Focusing on Remnant Pancreas Recurrence After Surgical Resection
}

\section{Tomokazu FUJI}

Okayama University Graduate School of Medicine Dentistry and Pharmaceutical Sciences Yuzo UMEDA ( $\sim$ y.umeda@d9.dion.ne.jp)

Okayama University Graduate School of Medicine Dentistry and Pharmaceutical Sciences Kosei TAKAGI

Okayama University Graduate School of Medicine Dentistry and Pharmaceutical Sciences Ryuichi YOSHIDA

Okayama University Graduate School of Medicine Dentistry and Pharmaceutical Sciences

\section{Kazuhiro YOSHIDA}

Okayama University Graduate School of Medicine Dentistry and Pharmaceutical Sciences

\section{Kazuya YASUI}

Okayama University Graduate School of Medicine Dentistry and Pharmaceutical Sciences

\section{Kazuyuki MATSUMOTO}

Okayama University Graduate School of Medicine Dentistry and Pharmaceutical Sciences

\section{Hironari KATO}

Okayama University Graduate School of Medicine Dentistry and Pharmaceutical Sciences

\section{Takahito YAGI}

Okayama University Graduate School of Medicine Dentistry and Pharmaceutical Sciences Toshiyoshi FUJIWARA

Okayama University Graduate School of Medicine Dentistry and Pharmaceutical Sciences

\section{Research Article}

Keywords: pancreatic intraductal neoplasms, pancreatectomy, recurrence

Posted Date: December 15th, 2021

DOI: https://doi.org/10.21203/rs.3.rs-1123860/v1

License: (a) (i) This work is licensed under a Creative Commons Attribution 4.0 International License. Read Full License 
Page $2 / 13$ 


\section{Abstract}

Background: The international consensus guidelines for intraductal papillary mucinous neoplasm of the pancreas (IPMN) presented clinical features as indications for surgery. Whereas surveillance for recurrence including de novo lesions is essential, optimal surveillance protocols have not been established.

Aim and Methods: The aim of this study was to assess the clinical features of recurrence at the remnant pancreas (Rem-Panc) and extra-pancreas (Ex-Panc) after surgery for IPMN. Ninety-one cases of IPMN that underwent detailed preoperative assessment and pancreatectomy were retrospectively analyzed, focusing especially on the type of recurrence.

Results: The IPMNs were finally diagnosed as low-grade dysplasia (LDA, $n=42)$, high-grade dysplasia (HAD, $n=19)$, and invasive carcinoma (IPMC, $n=30$ ). Recurrence was observed in 26 cases (28\%), of which recurrence was seen at Rem-Panc in 19 cases and at Ex-Panc in 7 cases. The frequency of Rem-Panc recurrence was $10 \%$ in LDA, $21 \%$ in HDA, and $37 \%$ in IPMC. On the other hand, Ex-Panc recurrence was observed only in IPMC (23\%). Ex-Panc recurrence showed shorter median recurrence-free survival (RFS) and overall survival (OS) than Rem-Panc recurrence (median RFS 8 months vs 35 months, $p<0.001$; median OS 25 months vs 72 months, $p<0.001)$. Regarding treatment for Rem-Panc recurrence, repeat pancreatectomy resulted in better OS than no-pancreatectomy (MST 36 months vs 15.5 months, $p=0.03$ ). On multivariate analysis, main duct stenosis or disruption as a preoperative feature (hazard ratio [HR] 10.6, $p=0.002)$ and positive surgical margin (HR 4.4, $p=0.018$ ) were identified as risk factors for Rem-Panc recurrence.

Conclusions: The risk factors for Rem-Panc and Ex-Panc recurrence differ. Optimal surveillance based on these features is desirable to ensure that repeat pancreatectomy for Rem-Panc recurrence can be an appropriate surgical intervention.

\section{Introduction}

For the clinical management of intraductal papillary mucinous neoplasms of the pancreas (IPMNs), an international consensus guideline was provided by the International Association of Pancreatology[1-3]. These guidelines for clinical management, including radiological and endoscopic follow-up and surgical indications, have been widely accepted. After surgical resection, the postoperative recurrence rate of IPMN was reported to $6.8-9.6 \%$ with non-invasive IPMN and 32.2-65\% with invasive IPMN[4-7]. Notably, IPMN recurrences have various temporospatial distributions[7]. Previous studies of postoperative recurrence sites of IPMN showed that distant metastasis occurred mostly in invasive IPMN. The form of recurrence depends on the differentiation of the primary IPMN; the recurrence rates were $0-7.7 \%$ with remnant pancreas recurrence (Rem-Panc), 0-1.3\% with extrapancreatic metastasis (Ex-Panc), and 0-0.8\% with both sites in non-invasive IPMN, whereas they were $5.7-15.0 \%, 35.0-45.7 \%$, and $2.9-8.8 \%$ in invasive cancer[4, 5, 8]. Perioperative risk factors for the recurrence pattern have been debated. Risk factors for Rem-Panc recurrence were reported to be presence of high-grade dysplasia in resected specimens, positive surgical 
margins, and family history of pancreatic ductal adenocarcinoma (PDAC). It was also suggested that the gastric or pancreatobiliary type as IPMN subtypes could be potential indicators of carcinogenic recurrence as PDAC in Rem-Panc $[9,10]$.

Several guidelines for IPMN refer to the risk of postoperative recurrence and the follow-up policy. For noninvasive IPMN, including low-grade and high-grade dysplasia, surveillance with a focus on the remnant pancreas is recommended, because early detection of a malignant lesion and surgical resection may improve the prognosis. The International Consensus Guideline 2017 advocates that cross-sectional imaging be required for high-risk factors for Rem-Panc recurrence, such as: family history of pancreatic cancer; non-invasive IPMN or invasive IPMC at the surgical margin; and non-intestinal subtypes [3]. However, in reality, the first two are relatively rare. In addition, the European Study Group On Cystic Tumors

of The Pancreas guidelines specify high-grade dysplasia and main duct type as high-risk factors [11], and the AGA guidelines do not recommend periodic screening for low-grade dysplasia (LGD), because of its lack of cost-effectiveness[12]. Thus, there has not been consensus in this field. From these points of view, this study aimed to assess the clinical features of IPMN recurrence.

\section{Materials And Methods}

\section{Study subjects}

A total of 91 cases of IPMN that underwent initial surgical resection with curative intent in Okayama University Hospital from May 2007 to December 2014 were retrospectively reviewed. Demographic information, symptoms at presentation, radiological and endoscopic findings, surgical procedures, pathology, and postoperative course were collected from medical records.

With regard to precise preoperative tumor assessment, all patients were assessed by endoscopic ultrasonography (EUS) using contrast enhancement for intraductal mural nodules and by pancreatic juice cytology under endoscopic retrograde cholangiopancreatography (ERCP). The diameter of the main pancreatic duct or branched cyst was measured by computed tomography (CT) or MRI. Definite mural nodules or pancreatic duct features such as stenosis or disruption were determined by these diagnostic modalities. Preoperative pancreatitis and obstructive jaundice were judged by clinical findings including laboratory or radiological imaging data.

\section{Surgical indications, pathological examination, and postoperative follow-up}

Indications for surgery were determined on the basis of international consensus guideline 2006[1] or 2012[2]. Patients with a preoperative diagnosis or highly suspicious of invasive cancer underwent pancreatectomy with regional lymph node dissection. Resected specimens were reviewed by pathologists and classified into 3 groups according to the World Health Organization (WHO) classification: IPMN lowgrade dysplasia (LGD), IPMN high-grade dysplasia (HGD), and IPMN-associated invasive carcinoma (IPMC). If different grades coexisted in one lesion, the highest degree of dysplasia was adopted as the classification. Surgical margins were examined at pancreatic transection margins and dissected 
peripancreatic tissue margins, which were classified as negative or positive for LGD, HGD, and IPMC. Final staging was based on these findings and the TNM classification in the seventh edition [13].

Adjuvant chemotherapy by gemcitabine or a fluorouracil-based agent for six months was given to the patients with a final pathological diagnosis of UICC-Stage 2. Postoperative follow-up with CT or MRI was performed according to the final pathology. Follow-up intervals varied by IPMN-classification: 6 to 12 months for LGD, 3 to 6 months for HGD, and 3 months for IPMC. If tumor recurrence or metastasis was suspected, further examinations including EUS, CT, and MRI were performed as needed.

Tumor recurrence was classified into two types. One type was Rem-Panc recurrence, including de novo IPMN, PDAC, and obvious enlargement of preexisting IPMN in the remnant pancreas. The diagnosis of Rem-Panc recurrence required endoscopic biopsy. The other type was extrapancreatic (Ex-Panc) recurrence, including metastases to liver, lung, lymph node, and peritoneum (Supplemental Figure 1).

\section{Statistical analysis}

Clinical variables were compared using the Mann-Whitney $U$ test for continuous data and Pearson's correlation coefficient for categorical data. Continuous variables are presented as medians and interquartile range (IQR). Values of $p<0.05$ were considered significant. Overall survival (OS), recurrencefree survival (RFS), and incidence of Rem-Panc recurrence were evaluated using the Kaplan-Meier method and compared using log-rank tests. Cox's proportional hazards model with clinical variables including high-risk stigmata and worrisome EUS features [2] was used to identify prognostic factors for Rem-Panc recurrence. For this analysis, clinical variables showing values of $p<0.05$ on univariate analyses were entered into the multivariate analysis. The event of Ex-Panc recurrence and other causes of death were treated as censored. Hazard ratios (HRs) and $95 \%$ confidence intervals (95\%Cls) were calculated. All statistical analyses were performed using JMP version 14 (SAS Institute Inc., Cary, NC, USA).

\section{Ethics approval and consent to participate}

This study conformed to the Declaration of Helsinki on Human Research Ethics standards and was approved by the Okayama University Hospital Institutional Ethics Board (number 1902-019). The need for written, informed consent was waived by the Okayama University Hospital Institutional Ethics Board because of the retrospective design.

\section{Results}

Clinical and pathological background characteristics of the enrolled patients are summarized in Table 1. As preoperative examinations, contrast-enhanced CT was performed in all patients, EUS in 89 patients (97.8\%), and pancreatic juice cytology in 78 patients (85.7\%). The most frequent pathological type was LGD $(n=42,46.2 \%)$, followed by IPMC $(n=30,33.0 \%)$ and HGD $(n=19,20.9 \%)$. Sixty cases $(65.9 \%)$ had negative surgical margins; the other 31 cases had positive surgical margins. These surgical margins were pathologically diagnosed as LGD $(n=22,24.2 \%), \operatorname{HGD}(n=6,6.6 \%)$, and IPMC $(n=3,3.3 \%)$. In three cases 
with positive margins as IPMC, postoperative diagnosis overturned the result, despite a negative margin on intra-operative frozen section examination. There was no hospital mortality. The median and mean follow-up periods after resection were 44 and 47.4 months, respectively (range, 1-106 months). In this follow-up period, tumor recurrence was confirmed in 26 patients, of whom 19 patients (20.9\%) showed Rem-Panc recurrence, and seven patients (7.7\%) showed Ex-Panc recurrence.

Ex-Panc recurrence was characterized by a higher CA19-9 level and higher rates of clinical symptoms, enhancing solid component, class 4/5 on pancreatic juice cytology, and lymph node metastasis than Rem-Panc recurrence. Ex-Panc recurrence was observed only in IPMC patients as the primary pathology $(n=\star \star, 23.3 \%)$. Distant metastatic sites in Ex-Panc recurrence were liver $(n=1)$, lung $(n=2)$, lymph nodes $(n=2)$, and peritoneum $(n=2)$. On the other hand, from the viewpoint of primary pathology, the frequency of Rem-Panc recurrence increased with pathological malignant grade; Rem-Panc recurrence occurred the most frequently in IPMC ( $n=12,36.7 \%)$, followed by HGD ( $n=15,21.1 \%)$ and LGD $(n=38,9.5 \%)$ (Figure 1).

Concerning recurrence and survival analysis, median and mean follow-up periods were 71 months and 72.7 months, respectively. IPMC had the shortest recurrence-free time, followed by HGD and LGD; $2 / 5 / 10$ year recurrence rates were $26 / 36 / 65 \%$ in IPMC, $6 / 18 / 26 \%$ in HGD, $0 / 0 / 16 \%$ in LGD, respectively $(p=0.001$ ) (Figure 2a). Multivariate analysis using perioperative parameters identified main duct stenosis or disruption (HR 10.63, $p=0.002$ ) and HGD/IPMC at the surgical margin (HR4.4; $p=0.018$ ) as independent risk factors for Rem-Panc recurrence (Table 2). Patients with one of these risk factors showed a significant potential for Rem-Panc recurrence compared to patients with no risk factors $(2 / 5 / 10$ year recurrence rates $27 / 56 / 64 \%$ vs $3 / 3 / 20 \%$, $p<0.001$ ) (Figure $2 b$ ).

Concerning RFS and OS, Ex-Panc recurrence resulted in a worse prognosis than Rem-Panc recurrence (median RFS 8 months vs 35 months, $p<0.0001$; median OS 25 months vs 72 months, $\mathrm{p}<0.0001$ ) (Figure 3a, 3b).

In patients with Rem-Panc recurrence $(n=19)$, repeat pancreatectomy was performed in 9 patients $(47.3 \%)$. Survival after Rem-Panc recurrence of patients treated by repeat pancreatectomy was better than that of patients without any surgical intervention (median OS 36 months vs 15.5 months, $p=0.03$ ) (Figure 4).

\section{Discussion}

International consensus guidelines suggest that there should be no time limit for follow-up after resection of IPMNs, but the specific surveillance protocol remains unclear [3]. The aim of this study was to clarify the optimal surveillance strategy according to risk factor analysis for Rem-Panc and Ex-Panc recurrence after resection. RFS and OS differed between the recurrence patterns: Ex-Panc recurrence tended to occur earlier in the postoperative period and to result in a poorer prognosis than Rem-Panc recurrence. In the comparison of clinical background characteristics between patients with intrapancreatic or extrapancreatic recurrence, as shown in Figure 2, all Ex-Panc recurrences occurred in primary IPMC cases. In addition, Ex-Panc recurrence showed a higher serum CA19-9 level, jaundice, and advanced UICC stage. Namely, Ex-Panc recurrence could rely heavily on primary tumor biology, and systemic screening during 
the early postoperative period would be preferable. Perhaps adjuvant chemotherapy or neoadjuvant chemotherapy should be considered in these cases.

On the other hand, Rem-Panc recurrence also depends on primary tumor biology. IPMC or HGD as primary pathology showed higher and earlier Rem-Panc recurrence than LGD. Furthermore, IPMC or HGD as a positive surgical margin could be an independent risk factor for Rem-Panc recurrence. On the basis of these results, the potential of field carcinogenesis of Rem-Panc would be reflected in the degree of differentiation of the primary tumor. Furthermore, the fact that LGD showed Rem-Panc recurrence even after 5 years suggests that surveillance of Rem-Panc after resection of IPMN should be continued throughout the patient's lifetime, regardless of tumor grading. Regarding post-Rem-Panc recurrence survival, only repeated surgery could be a curative treatment option that would contribute to long-term survival. The main reason why 10 cases of Rem-Panc recurrence did not have the opportunity for repeat pancreatectomy was locally advanced Rem-Panc IPMC or ductal cell carcinoma with distant metastasis, because of the delayed diagnosis of recurrent disease.

As the present results show, about half of the patients with risk-positive disease develop Rem-Panc recurrence within 5 years after surgery, suggesting that repeated imaging checks each year, especially focusing on the pancreatic duct by MRCP, are recommended for early detection of Rem-Panc recurrence. Hirono reported recurrence patterns and risk factors after surgical resection of 1,074 IPMNs as a project study of the Japan Pancreas Society[14]. In this analysis, recurrence type was classified into "High-risk lesions in the remnant pancreas" and "Extrapancreatic recurrence". Preoperative clinical symptoms, pancreatic body/tail as the IPMN location, main duct size $>10 \mathrm{~mm}$, and HGD/invasive IPMC were identified as independent risk factors for "High-risk lesions in the remnant pancreas". In addition to these findings, we first identified that preoperative EUS findings could be an important predictor of Rem-Panc recurrence after IPMN resection, including HGD/IPMC at the surgical margin and main duct stenosis or disruption as a preoperative EUS feature. According to previous studies, there are contradictory opinions about the surgical margin. It has been reported that a positive margin is associated with the risk of postoperative recurrence[15] [16], whereas another paper showed there was no relationship[17] [18]. Interestingly, Frankel reported that dysplasia at the margin after pancreatectomy for non-invasive IPMN is associated with recurrence in the remnant gland [19]. They considered that, when dysplasia is present at multiple locations within the pancreas, such as the surgical margin and/or extracystic duct, patients are at increased risk of developing recurrent IPMN, supporting the concept of a 'field defect'. Knowledge about the correlation between recurrence risk and stenosis/disruption of the main pancreatic duct has been insufficient. Pea advocated three different mechanisms to explain the development of malignant lesions in the residual pancreas after IPMN surgery[20]: (1) tumor resection at the surgical margin of the pancreas; (2) spread of tumor cells into pancreatic ducts or parenchyma; and (3) independent multifocal lesions. The features of the main pancreatic duct might suggest tumor spread into remnant pancreatic parenchyma as in the second mechanism. In general, most studies did not report any major difference in the risk of obstructive pancreatitis between benign and malignant IPMNs [21-23], because mucous embolism of the main pancreatic duct is more frequent in intestinal type with a better 
prognosis $[24,25]$. In addition, the maximum diameter of the main pancreatic duct was correlated with the distance of tumor spread in the main pancreatic duct [26].

Consequently, a specific surveillance protocol for IPMN should be stratified, focusing on two different types of recurrence, since a half of patients with a high risk of Rem-Panc recurrence would recur within 5 years after surgery. Therefore, Rem-Panc patients should be closely checked by various diagnostic modalities, including EUS or MRCP $[27,28]$. Even in patients without any risk factors, the possibility of Rem-Panc recurrence persists throughout the patients' lifetime; thus, uninterrupted surveillance is necessary. On the other hand, Ex-Panc recurrence was characterized only by IPMC as the primary lesion and would recur within two years. Careful follow-up 3-4 times a year for 2 years after surgery is desirable, and selection of neo-adjuvant and adjuvant chemotherapy in addition to surgery is an issue for further study.

Some limitations of our study were that it was a retrospective study at a single center, and the surveillance strategy was not standardized throughout the follow-up period. Nonetheless, despite these limitations, the following follow-up strategy might be considered based on the risk factors for Rem-Panc or Ex-Panc.

\section{Conclusion}

The present study showed the possibility of IPMN stratification by risk according to type of relapse using perioperative factors for the treatment strategy. IPMC cases might need systemic surveillance focusing on extrapancreatic recurrence within two years after primary pancreatectomy. On the other hand, in any type of IPMN, remnant pancreatic screening is necessary for life, and interval screening depends on risk factors.

\section{Declarations}

\section{Ethics approval and consent to participate}

This study conformed to the Declaration of Helsinki on Human Research Ethics standards and was approved by the Okayama University Hospital Institutional Ethics Board (number 1902-019). The need for written, informed consent was waived by the Okayama University Hospital Institutional Ethics Board because of the retrospective design.

\section{Consent for publication}

Not applicable

\section{Availability of data and materials}

The datasets generated and/or analyzed during the current study are not publicly available due owing to data privacy policy at our facility, but are available from the corresponding author on reasonable request. 
Conflict of interest

None

\section{Authors' contributions}

Tomokazu FUJl: writing

Yuzo UMEDA: Study design, data analysis, and writing

Ryuichi YOSHIDA, Kazuhiro YOSHIDA, Kosei TAKAGI, Kazuya YASUI, Kazuyuki MATSUMOTO, Hironari KATO, Takahito YAGI, and Toshiyoshi FUJIWARA: data collection

Funding support: None

Acknowledgement: Not applicable

\section{References}

1. Tanaka M, Chari S, Adsay V, Fernandez-del Castillo C, Falconi M, Shimizu M, Yamaguchi K, Yamao K, Matsuno S, International Association of P: International consensus guidelines for management of intraductal papillary mucinous neoplasms and mucinous cystic neoplasms of the pancreas. Pancreatology: official journal of the International Association of Pancreatology 2006, 6(1-2):17-32.

2. Tanaka M, Fernandez-del Castillo C, Adsay V, Chari S, Falconi M, Jang JY, Kimura W, Levy P, Pitman MB, Schmidt CM et al: International consensus guidelines 2012 for the management of IPMN and MCN of the pancreas. Pancreatology: official journal of the International Association of Pancreatology 2012, 12(3):183-197.

3. Tanaka M, Fernandez-Del Castillo C, Kamisawa T, Jang JY, Levy P, Ohtsuka T, Salvia R, Shimizu Y, Tada M, Wolfgang CL: Revisions of international consensus Fukuoka guidelines for the management of IPMN of the pancreas. Pancreatology: official journal of the International Association of Pancreatology 2017, 17(5):738-753.

4. Chari ST, Yadav D, Smyrk TC, DiMagno EP, Miller LJ, Raimondo M, Clain JE, Norton IA, Pearson RK, Petersen BT et al: Study of recurrence after surgical resection of intraductal papillary mucinous neoplasm of the pancreas. Gastroenterology 2002, 123(5):1500-1507.

5. Schnelldorfer T, Sarr MG, Nagorney DM, Zhang L, Smyrk TC, Qin R, Chari ST, Farnell MB: Experience with 208 resections for intraductal papillary mucinous neoplasm of the pancreas. Archives of surgery 2008, 143(7):639-646; discussion 646.

6. Winner M, Epelboym I, Remotti H, Lee JL, Schrope BA, Chabot JA, Allendorf JD: Predictors of recurrence in intraductal papillary mucinous neoplasm: experience with 183 pancreatic resections. Journal of gastrointestinal surgery: official journal of the Society for Surgery of the Alimentary Tract 2013, 17(9):1618-1626. 
7. Marchegiani G, Mino-Kenudson M, Ferrone CR, Morales-Oyarvide V, Warshaw AL, Lillemoe KD, Castillo CF: Patterns of Recurrence After Resection of IPMN: Who, When, and How? Annals of surgery 2015, 262(6):1108-1114.

8. Yogi T, Hijioka S, Imaoka H, Mizuno N, Hara K, Tajika M, Tanaka T, Ishihara M, Shimizu Y, Hosoda W et al: Risk factors for postoperative recurrence of intraductal papillary mutinous neoplasms of the pancreas based on a long-term follow-up study: proposals for follow-up strategies. Journal of Hepato-Biliary-Pancreatic Sciences 2015, 22(10):757-765.

9. Ideno N, Ohtsuka T, Matsunaga T, Kimura H, Watanabe Y, Tamura K, Aso T, Aishima S, Miyasaka Y, Ohuchida $\mathrm{K}$ et al: Clinical significance of GNAS mutation in intraductal papillary mucinous neoplasm of the pancreas with concomitant pancreatic ductal adenocarcinoma. Pancreas 2015, 44(2):311320.

10. Miyasaka Y, Ohtsuka T, Tamura K, Mori Y, Shindo K, Yamada D, Takahata S, Ishigami K, Ito T, Tokunaga S et al: Predictive Factors for the Metachronous Development of High-risk Lesions in the Remnant Pancreas After Partial Pancreatectomy for Intraductal Papillary Mucinous Neoplasm. Annals of surgery 2016, 263(6):1180-1187.

11. European Study Group on Cystic Tumours of the P: European evidence-based guidelines on pancreatic cystic neoplasms. Gut 2018, 67(5):789-804.

12. Vege SS, Ziring B, Jain R, Moayyedi P, Clinical Guidelines C, American Gastroenterology A: American gastroenterological association institute guideline on the diagnosis and management of asymptomatic neoplastic pancreatic cysts. Gastroenterology 2015, 148(4):819-822; quize812-813.

13. American Joint Committee on C, Edge SB: AJCC cancer staging manual, 7th ed edn: Springer; 2010.

14. Hirono S, Shimizu Y, Ohtsuka T, Kin T, Hara K, Kanno A, Koshita S, Hanada K, Kitano M, Inoue H et al: Recurrence patterns after surgical resection of intraductal papillary mucinous neoplasm (IPMN) of the pancreas; a multicenter, retrospective study of 1074 IPMN patients by the Japan Pancreas Society. Journal of gastroenterology 2020, 55(1):86-99.

15. Leng KM, Wang ZD, Zhao JB, Cui YF, Zhong XY: Impact of pancreatic margin status and lymph node metastases on recurrence after resection for invasive and noninvasive intraductal papillary mucinous neoplasms of the pancreas: a meta-analysis. Digestive surgery 2012, 29(3):213-225.

16. White R, D'Angelica M, Katabi N, Tang L, Klimstra D, Fong Y, Brennan M, Allen P: Fate of the remnant pancreas after resection of noninvasive intraductal papillary mucinous neoplasm. Journal of the American College of Surgeons 2007, 204(5):987-993; discussion 993-985.

17. Fujii T, Kato K, Kodera Y, Kanda M, Nagai S, Yamada S, Kanzaki A, Sugimoto H, Nomoto S, Takeda S et al: Prognostic impact of pancreatic margin status in the intraductal papillary mucinous neoplasms of the pancreas. Surgery 2010, 148(2):285-290.

18. Tamura K, Ohtsuka T, Ideno N, Aso T, Shindo K, Aishima S, Ohuchida K, Takahata S, Ushijima Y, Ito T et al: Treatment strategy for main duct intraductal papillary mucinous neoplasms of the pancreas based on the assessment of recurrence in the remnant pancreas after resection: a retrospective review. Annals of surgery 2014, 259(2):360-368. 
19. Frankel TL, LaFemina J, Bamboat ZM, D'Angelica MI, DeMatteo RP, Fong Y, Kingham TP, Jarnagin WR, Allen PJ: Dysplasia at the surgical margin is associated with recurrence after resection of noninvasive intraductal papillary mucinous neoplasms. HPB: the official journal of the International Hepato Pancreato Biliary Association 2013, 15(10):814-821.

20. Pea A, Yu J, Rezaee N, Luchini C, He J, Dal Molin M, Griffin JF, Fedor H, Fesharakizadeh S, Salvia R et al: Targeted DNA Sequencing Reveals Patterns of Local Progression in the Pancreatic Remnant
Following Resection of Intraductal Papillary Mucinous Neoplasm (IPMN) of the Pancreas. Annals of
surgery 2016.

21. Rivera JA, Fernandez-del Castillo C, Pins M, Compton CC, Lewandrowski KB, Rattner DW, Warshaw AL: Pancreatic mucinous ductal ectasia and intraductal papillary neoplasms. A single malignant clinicopathologic entity. Annals of surgery 1997, 225(6):637-644; discussion 644-636.

22. Pelletier AL, Hammel P, Rebours V, Couvelard A, Vullierme MP, Maire F, Hentic O, Aubert A, Sauvanet A, Levy $\mathrm{P}$ et al: Acute pancreatitis in patients operated on for intraductal papillary mucinous neoplasms of the pancreas: frequency, severity, and clinicopathologic correlations. Pancreas 2010, 39(5):658661.

23. Venkatesh PG, Navaneethan U, Vege SS: Intraductal papillary mucinous neoplasm and acute pancreatitis. Journal of clinical gastroenterology 2011, 45(9):755-758.

24. Marchegiani G, Mino-Kenudson M, Sahora K, Morales-Oyarvide V, Thayer S, Ferrone C, Warshaw AL, Lillemoe KD, Fernandez-Del Castillo C: IPMN involving the main pancreatic duct: biology, epidemiology, and long-term outcomes following resection. Annals of surgery 2015, 261(5):976-983.

25. Saito M, Imada H, Suzuki T, Sata N, Yasuda Y, Maetani I, Fukushima N: Distinct patterns of peritumoral histological findings in subtypes of intraductal papillary mucinous neoplasms of the pancreas. Annals of diagnostic pathology 2015, 19(5):347-352.

26. Okada K-i, Imaizumi T, Hirabayashi K, Matsuyama M, Yazawa N, Dowaki S, Tobita K, Ohtani Y, Kawaguchi Y, Tanaka M et al: The distance of tumor spread in the main pancreatic duct of an intraductal papillary-mucinous neoplasm: where to resect and how to predict it. Journal of HepatoBiliary-Pancreatic Sciences 2010, 17(4):516-522.

27. Kawakami S, Fukasawa M, Shimizu T, Ichikawa S, Sato T, Takano S, Kadokura M, Shindo H, Takahashi E, Hirose S et al: Diffusion-weighted image improves detectability of magnetic resonance cholangiopancreatography for pancreatic ductal adenocarcinoma concomitant with intraductal papillary mucinous neoplasm. Medicine 2019, 98(47):e18039.

28. Kamata K, Takenaka M, Minaga K, Omoto S, Miyata T, Yamao K, Imai H, Nakai A, Tanaka H, Chiba Y et al: Value of additional endoscopic ultrasonography for surveillance after surgical removal of intraductal papillary mucinous neoplasms. Digestive endoscopy: official journal of the Japan Gastroenterological Endoscopy Society 2018, 30(5):659-666.

29. Strauss A, Birdsey M, Fritz S, Schwarz-Bundy BD, Bergmann F, Hackert T, Kauczor HU, Grenacher L, Klauss M: Intraductal papillary mucinous neoplasms of the pancreas: radiological predictors of malignant transformation and the introduction of bile duct dilation to current guidelines. The British journal of radiology 2016, 89(1061):20150853. 
30. Maker AV, Katabi N, Gonen M, DeMatteo RP, D'Angelica MI, Fong Y, Jarnagin WR, Brennan MF, Allen PJ: Pancreatic cyst fluid and serum mucin levels predict dysplasia in intraductal papillary mucinous neoplasms of the pancreas. Annals of surgical oncology 2011, 18(1):199-206.

31. Mukewar S, de Pretis N, Aryal-Khanal A, Ahmed N, Sah R, Enders F, Larson JJ, Levy MJ, Takahashi N, Topazian $\mathrm{M}$ et al: Fukuoka criteria accurately predict risk for adverse outcomes during follow-up of pancreatic cysts presumed to be intraductal papillary mucinous neoplasms. Gut 2016.

32. Aso T, Ohtsuka T, Matsunaga T, Kimura H, Watanabe $Y$, Tamura $K$, Ideno N, Osoegawa T, Takahata S, Shindo $\mathrm{K}$ et al: "High-risk stigmata" of the $\mathbf{2 0 1 2}$ international consensus guidelines correlate with the malignant grade of branch duct intraductal papillary mucinous neoplasms of the pancreas. Pancreas 2014, 43(8):1239-1243.

33. Monnings P, Belyaev O, Uhl W, Giese A, Tannapfel A, Koster O, Meier JJ: Criteria for Determining Malignancy in Pancreatic Intraductal Papillary Mucinous Neoplasm Based on Computed Tomography. Digestion 2016, 94(4):230-239.

\section{Tables}

Due to technical limitations, table 1 and 2 is only available as a download in the Supplemental Files section.

\section{Figures}

\section{Figure 1}

Correlations between the pathological grade of the resected IPMN lesion and recurrence patterns.

\section{Figure 2}

(a) Cumulative recurrence rate at the remnant pancreas, stratified by primary tumor grading (b) Cumulative recurrence rate at the remnant pancreas, stratified by risk factor

\section{Figure 3}

(a) Recurrence-free survival curves after resection, stratified by recurrence pattern. (b) Overall-survival curves after resection, stratified by recurrence pattern. 


$\begin{array}{llll}\text { - Repeat pancreatectomy }(\mathrm{n}=9) & 78 \% & 39 \% \\ \text { - No repeat pancreatectomy }(\mathrm{n}=7) & 40 \% & 10 \%\end{array}$

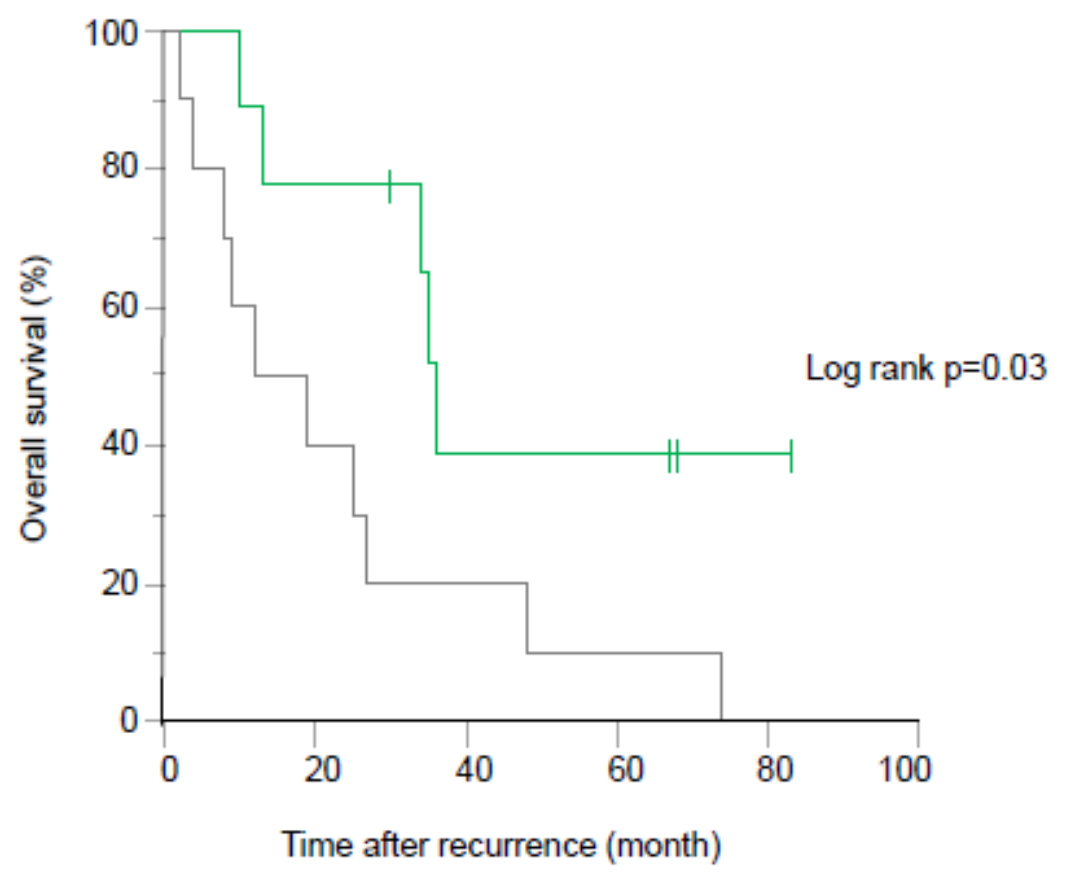

Number at Risk

$\begin{array}{lcccccc}\text { Repeat pancreatectomy } & 9 & 7 & 3 & 3 & 1 & 0 \\ \text { No repeat pancreatectomy } & 10 & 4 & 2 & 1 & 0 & 0\end{array}$

Figure 4

Overall survival curves after remnant pancreatic recurrence, comparing patients with and without repeat pancreatectomy.

\section{Supplementary Files}

This is a list of supplementary files associated with this preprint. Click to download.

- R2106933Table11.xlsx

- R2106933Table21.xlsx

- supplementalFigure1.pdf

- supplementalFigure2.pdf 